Article

\title{
Quantum Bits with Macroscopic Topologically Protected States in Semiconductor Devices
}

\author{
Błażej Jaworowski 1,2,*(D) and Paweł Hawrylak ${ }^{2}$ \\ 1 Department of Theoretical Physics, Wrocław University of Science and Technology, Wybrzeże \\ Wyspiańskiego 27, 50-370 Wrocław, Poland \\ 2 Department of Physics, University of Ottawa, Ottawa, ON K1N 6N5, Canada; Pawel.Hawrylak@uottawa.ca \\ * Correspondence: blazej.jaworowski@pwr.edu.pl
}

Received: 22 December 2018; Accepted: 25 January 2019; Published: 30 January 2019

\begin{abstract}
Current computers are made of semiconductors. Semiconductor technology enables realization of microscopic quantum bits based on electron spins of individual electrons localized by gates in field effect transistors. This results in very fragile quantum processors prone to decoherence. Here, we discuss an alternative approach to constructing qubits using macroscopic and topologically protected states realized in semiconductor devices. First, we discuss a synthetic spin- 1 chain realized in an array of quantum dots in a semiconductor nanowire or in a field effect transitor. A synthetic spin-1 chain is characterized by two effective edge quasiparticles with spin $1 / 2$ protected from decoherence by topology and Haldane gap. The spin-1/2 quasiparticles of Haldane phase form the basis of a macroscopic singlet-triplet qubit. We compare the spin one chain with a Kitaev chain. Its edge states are Majorana zero modes, possessing non-Abelian fractional statistics. They can be used to encode the quantum information using the braiding processes, i.e., encircling one particle by another, which do not depend on the details of the particle trajectory and thus are protected from decoherence.
\end{abstract}

Keywords: quantum computing; topological phases of matter; Haldane phase; Majorana fermions

\section{Introduction}

There are numerous proposals on how to realize qubits and quantum computers in a solid-state setup. For example, a spin- $1 / 2$ is a model two-state system, hence one option involves using the spin of a single electron within a quantum dot as a qubit [1-4]. The quantum information can be also encoded in states of several spins, belonging to decoherence-free subspaces [5-8]. An example is the singlet-triplet qubit, where the two-level system consists of $S^{z}=0$ singlet and triplet combinations of two spins $[7,8]$. Other proposals making use of microscopic states involve, for example, the nitrogen-vacancy centers in diamond [9,10], ionized donors [11] and nuclear spins [12] in silicon.

A different approach is to start with macroscopic quantum states. Some of the most robust and scalable qubits so far have been constructed using superconductors [13,14]. Another approach is to encode quantum information using the quasiparticles with non-Abelian fractional statistics [15]. The fractional (anyonic) statistics means that the particles are neither bosons nor fermions [16,17], while the non-Abelian nature is related to the fact that the exchange of two particle positions leads to mixing of several degenerate ground states [18]. Such situation can only occur in two dimensions [16] and is connected with the physics of topological phases of matter [19-21]. These phases cannot be described within the Landau paradigm of phase transitions, where a phase of matter is defined using a local order parameter measuring the degree to which a certain symmetry is broken [22,23]. In topological phases, there is no broken symmetry, and the phases are characterized by global topological invariants. Another distinctive feature of topological phases is the presence of edge states, which are robust to disorder. The quasiparticles with non-Abelian statistics are predicted to exist as excitations of fractional 
quantum Hall states [24,25], as well as edge states of one-dimensional topological superconductors [26]. It was proposed that the latter can be created, for example, by putting a semiconducting nanowire with strong spin-orbit coupling close to a superconductor $[27,28]$. The topological quantum computation, i.e., the quantum computation using anyons, proposed by Kitaev, is based on braiding operations [15]. Braiding means encircling one quasiparticle with another one. The effect of such operation does not depend on the exact trajectory of quasiparticles, so it should be insensitive to noise. Using the braiding operations, one can represent the quantum gates and assemble quantum circuits [29-31]. This proposal has numerous advantages. However, despite some observations consistent with the presence of fractional statistics [32], there is still no definite experimental evidence of their existence.

In addition to Kitaev's proposal, there are also ideas for utilizing the topological phases of matter for quantum computation in a way which does not involve fractional statistics [33-38]. While the non-Abelian anyons exist only in certain phases, all topological phases have robust edge states [19]. For example, the Haldane phase, occurring in antiferromagnetic spin-1 chains (e.g., in certain quasi-1D compounds [39-41]), is characterized by edge states behaving like effective spins-1/2 [42-44]. We proposed that an artificial, synthetic, semiconductor system, constructed with quantum dots, can be used to mimic such a chain $[45,46]$. Then, the edge spins can be used to create a macroscopic version of the singlet-triplet qubit, protected from the influence of disorder by the topological nature of the Haldane phase [45]. Such a qubit would combine the advantages of superconductor and semiconductor qubits: it is macroscopic, but on the other hand should be easily integrable with other semiconductor devices. Moreover, in principle, it should be possible to read such a qubit out using optical methods as well as convert it into a photon.

In this work, we review two examples of qubits based on the edge states of one-dimensional topological phases. First, we present our design based on a spin-1 chain. We review the origin of Haldane phase and its basic properties. Then, we show the principles of operation of our qubit and analyze two kinds of semiconductor systems which can be used to create it. Next, we review the topological quantum qubits based on the Majorana modes of a Kitaev chain [26]. Finally, we compare these two kinds of qubits, showing their similarities and differences, as well as strengths and weaknesses of each approach.

\section{Quantum Bits Based on Macroscopic Haldane Chains in Synthetic Semiconductor Systems}

\subsection{Haldane Phase in Synthetic Spin Chains}

We start with the properties of a Haldane phase in spin chains, a paradigm of topological quantum matter [42-44]. The Haldane phase exists in a one-dimensional spin-1 antiferromagnetic chain, described by the spin-1 Heisenberg model,

$$
H=J \sum_{i=1}^{N} \mathbf{S}_{i} \mathbf{S}_{i+1},
$$

where $J>0$ is the exchange constant, $\mathbf{S}_{i}$ is the spin- 1 operator at site $i$, and $N$ is the number of sites. Haldane has shown that such a chain has a gapped ground state in contrast to gapless spin- $1 / 2$ chains. [42-44]. In the case of a closed chain, this ground state is a nondegenerate singlet state. If the chain has open ends, then there are four degenerate ground states: a singlet and three triplets. The degeneracy is perfect in the thermodynamic limit, while, in a finite system, there is some splitting between singlet and triplet. Figure 1a shows the energy spectrum of the Heisenberg spin-1 chain, obtained using the exact diagonalization method [45]. The singlet and triplet alternate as ground states, but the splitting between them decreases as the chain length increases. The gap between these states and the quintuplet, however, remains finite, and converges to the black line, the Haldane gap, obtained by S. R. White using density matrix renormalization group method [47]. 

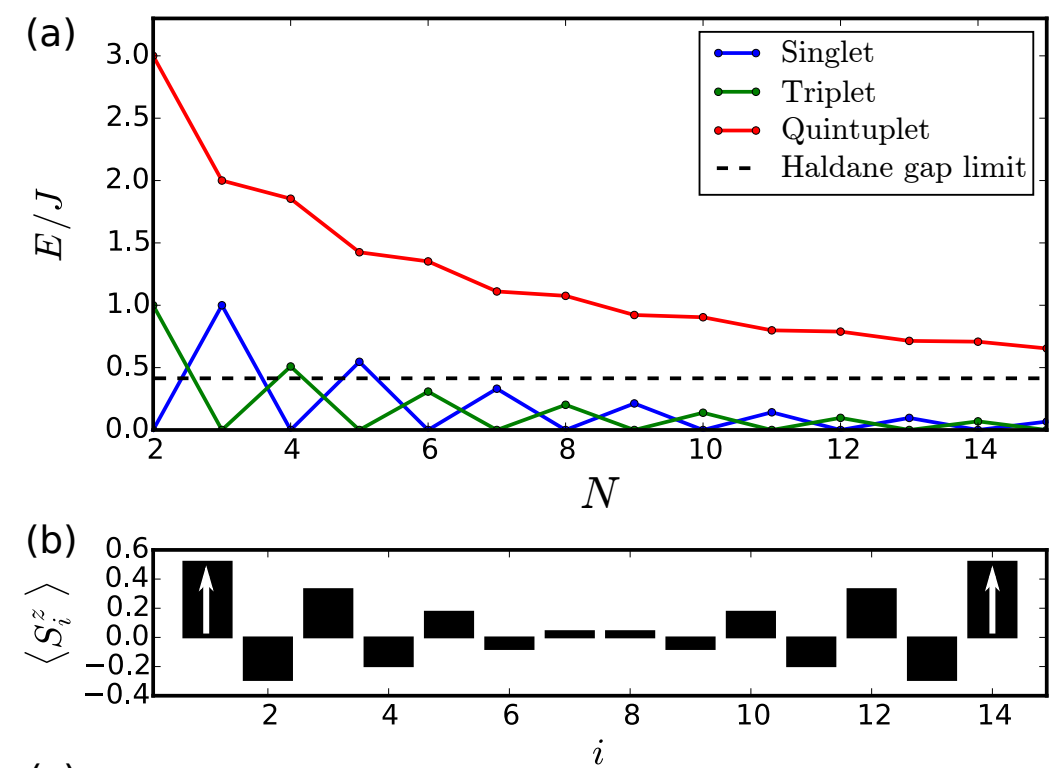

(c)

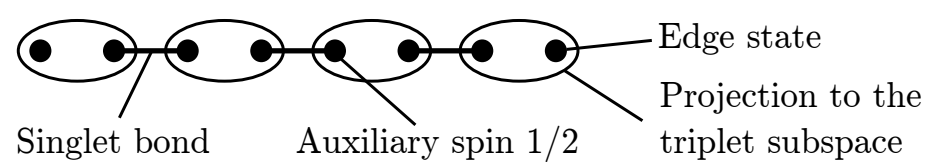

Figure 1. The Haldane phase. (a) the low-energy spectrum of spin-1 chains as a function of chain length $N$, obtained using exact diagonalization; (b) average spin density per site in $S^{z}=1$ triplet ground state of a $N=14$ chain. The white arrows symbolize the effective spins- $1 / 2$, indicated by spin density close to $1 / 2$ at the edges. (c) The Affleck-Kenedy-Lieb-Tasaki (AKLT) construction of the ground state.

The four ground states can be understood as superpositions of effective spins- $1 / 2$ located at the edges. Their existence can be seen in the site-resolved spin density in the the $S^{z}=1$ ground state (Figure $1 \mathrm{~b}$ ), being close to $1 / 2$ at the edges, and decreasing towards the middle of the chain. The finite spin density in the middle signifies an overlap of edge spins and thus a finite splitting between singlet and triplet.

The physical origin of the edge spins can be explained within the AKLT (Affleck, Kennedy, Lieb and Tasaki) model [48], a modified Heisenberg model defined by

$$
H_{\mathrm{AKLT}}=J \sum_{i}\left(\mathbf{S}_{i} \cdot \mathbf{S}_{i+1}+\frac{1}{3}\left(\mathbf{S}_{i} \cdot \mathbf{S}_{i+1}\right)^{2}+\frac{2}{3}\right) .
$$

Affleck et al. [48] have shown that this Hamiltonian can be written as a sum of a projection operator for nearest neighbor pairs of spins $P_{i}^{S=2}=\frac{1}{2} \mathbf{S}_{i} \cdot \mathbf{S}_{i+1}+\frac{1}{6}\left(\mathbf{S}_{i} \cdot \mathbf{S}_{i+1}\right)^{2}+\frac{1}{3}$ on the spin-2 subspace of this pair of spins. Thus, if there is a zero-energy ground state, it has no spin- 2 contribution on any such pair.

A prescription for creating such a state is given by Affleck et al. [48]. The key element is that a spin- 1 site $i$ can be considered as a triplet subspace of two spins-1/2, $i A$ and $i B$. To construct a ground state $\left|\psi_{\text {phys }}\right\rangle$, one can consider an auxiliary system, in which each spin- 1 is replaced by two spins- $1 / 2$, and then project its state $\left|\psi_{\text {aux }}\right\rangle$ to spins-1,

$$
\left|\psi_{\text {phys }}\right\rangle=\prod_{i} P_{\mathrm{AKLT}, i}\left|\psi_{\mathrm{aux}}\right\rangle,
$$

where

$$
P_{\mathrm{AKLT}, i}=P_{\mathrm{AKLT}, i}^{[+]}+P_{\mathrm{AKLT}, i}^{[-]}+P_{\mathrm{AKLT}, i}^{[0]},
$$


with

$$
\begin{aligned}
& P_{\mathrm{AKLT}, i}^{[+]}=\left|++_{i}\right\rangle\left\langle\uparrow_{i A} \uparrow_{i B}\right|, \\
& P_{\mathrm{AKLT}, i}^{[-]}=\left|-{ }_{i}\right\rangle\left\langle\downarrow_{i A} \downarrow_{i B}\right|, \\
& P_{\mathrm{AKLT}, i}^{[0]}=\frac{1}{\sqrt{2}}\left|0_{i}\right\rangle\left(\left\langle\uparrow_{i A} \downarrow_{i B}\right|+\left\langle\downarrow_{i A} \uparrow_{i B}\right|\right),
\end{aligned}
$$

where $\left|-{ }_{i}\right\rangle,\left|0_{i}\right\rangle,\left|+_{i}\right\rangle$ denote the $S^{z}=-1,0,1$ states of site $i$, respectively, while $\downarrow_{i \sigma}, \uparrow_{i \sigma}(\sigma=A, B)$ denote the $S^{z}=+1 / 2,-1 / 2$ states of auxiliary spin $i \sigma$, respectively.

It can be shown that $\left|\psi_{\text {phys }}\right\rangle$ has a spin-2 contribution if and only if $\left|\psi_{\text {aux }}\right\rangle$ has such a contribution. In Affleck et al. [48], it was shown that a desired $\left|\psi_{\text {aux }}\right\rangle$ consists of pairs of spins- $1 / 2$ on nearby sites connected by singlet bonds $\left|\psi_{i}^{\text {sing }}\right\rangle=\frac{1}{\sqrt{2}}\left(\left|\uparrow_{i, B} \downarrow_{i+1, A}\right\rangle-\left|\downarrow_{i, B} \uparrow_{i+1, A}\right\rangle\right)$ (see Figure $1 \mathrm{~b}$ ). This leaves two edge spins- $1 / 2$ unpaired, thus they can be in any state. As a consequence, there appear four zero-energy states, each of which can be understood as a given configuration of the edge spins. Note that, in contrast to the ordinary Heisenberg model, the degeneracy is perfect even for a finite system.

The Haldane phase belongs to a class of symmetry protected topological phases [49]. That is, it is robust to disorder, but only if a certain symmetry is obeyed. In the case of Haldane phase, this has to be at least one of the three symmetries: the $\pi$-rotation symmetry with respect to $x, y$ and $z$ axes, the time-reversal symmetry, and the reflection symmetry with respect to bond center $[49,50]$. All the systems connected adiabatically to the AKLT model, i.e., in which the Hamiltonian can be continuously deformed into AKLT Hamiltonian without closing the energy gap above the four ground states, and without breaking all three symmetries at once, belong to the Haldane phase.

\subsection{Macroscopic Singlet-Triplet Qubits}

Now, we will review our proposal for the application of the Haldane phase in quantum computing $[45,46]$, focusing on the general idea. The implementation of the spin chain in a solid-state system will be discussed later.

We start from considering the Heisenberg Hamiltonian with additional Zeeman terms describing the influence of external magnetic field: the uniform background field $B_{\mathrm{bg}}$ and a local field $B_{1}$ acting only on the first site,

$$
H_{\text {Zeeman }}=J \sum_{i=1}^{N-1} \mathbf{S}_{i} \cdot \mathbf{S}_{i+1}+g \mu B_{\mathrm{bg}} S_{\mathrm{tot}}^{z}+g \mu B_{1} S_{1}^{z}
$$

where $g$ is the Landé factor, $\mu$ is the Bohr magneton, $S_{\text {tot }}^{z}$ is the $z$ component of the total spin and $S_{1}^{z}$ is the $z$ component of spin of the first site.

As an example, we choose a chain of length $N=14$, in which the singlet and triplet states are well separated from the rest of the spectrum, but, on the other hand, there is still a noticeable splitting between these states, which will allow us to differentiate between the qubit states. The application of external field $B_{\mathrm{bg}}$ separates two $S^{z}= \pm 1$ states from $S^{z}=0$ ones (see Figure 2a). We choose these two states, the singlet $\left|S_{0}\right\rangle$ and $S^{z}=0$ triplet $\left|T_{0}\right\rangle$, as the two qubit states, similarly to what occurs in a conventional singlet-triplet qubit $[7,8]$. Note that the magnetic field cannot be too high because one should avoid the quintuplet states getting too close to the two qubit states. In the following analysis, we fix $\mu g B_{\mathrm{bg}} / J=0.17$, denoted by black line in Figure 2a.

To rotate the qubit state, one can use the local magnetic field $B_{1}$. The evolution of the energy spectrum with increasing $B_{1}$ is shown in Figure $2 \mathrm{~b}$. For $B_{1} \neq 0$, we call the qubit states $\left|a_{0}\right\rangle$ and $\left|a_{1}\right\rangle$, to distinguish them from the states $\left|S_{0}\right\rangle$ and $\left|T_{0}\right\rangle$ at $B_{1}=0$. It can be seen that these energy levels repel each other when the field is introduced. This is analogous to the case of two coupled spins- $1 / 2$ with a local magnetic field. We write $\left|a_{i}\right\rangle=A_{0}^{i}\left|S_{0}\right\rangle+A_{1}^{i}\left|T_{0}\right\rangle$. The components $A_{0}^{1}$ and $A_{1}^{1}$ of $\left|a_{1}\right\rangle$ are plotted in Figure 2c, showing the rotation of the qubit state as $B_{1}$ increases. We have verified that 
the admixture of states other than $\left|S_{0}\right\rangle$ and $\left|T_{0}\right\rangle$ is very small, at most $1.2 \%$, showing that the qubit subspace is well isolated from the rest of the spectrum.

Having shown the principles of operation of our qubit, let us move to its physical implementation. The existing experimental realization of spin-1 antiferromagnetic chains consist of quasi-1D compounds, which contain a number of parallel, weakly coupled chains [39-41]. However, we want to construct our synthetic spin one chain hosting a macroscopic quantum state using semiconductor technology. In the following subsections, we discuss two such examples.
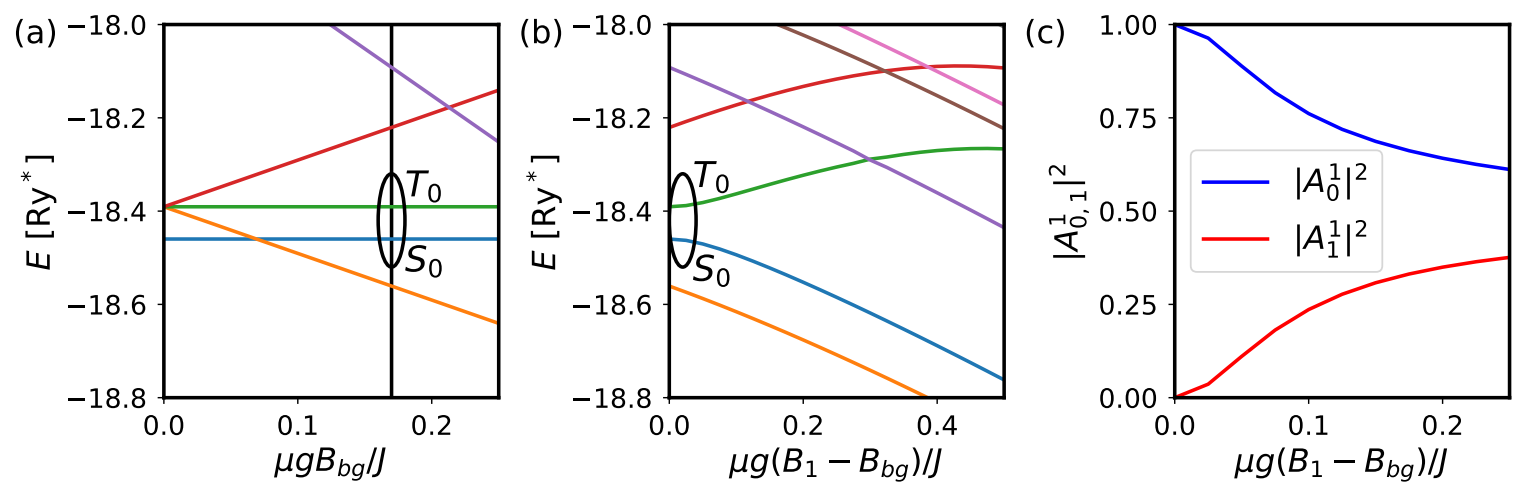

Figure 2. The idea of the singlet-triplet qubit based on the effective spin-1 chain. (a) the evolution of the energy spectrum of $n=14$ chain with increasing background magnetic field $B_{\mathrm{bg}}$, allowing for isolating the two levels $\left|S_{0}\right\rangle$ and $\left|T_{0}\right\rangle$. The vertical black line denotes $\mu g B_{\mathrm{bg}} / J=0.17 ;(\mathbf{b})$ the energy spectrum as a function of the local magnetic field $B_{1}$ on the leftmost dot. The singlet and triplet states mix, forming states $\left|a_{i}\right\rangle=A_{0}^{i}\left|S_{0}\right\rangle+A_{1}^{i}\left|T_{0}\right\rangle$, with $i=0,1 ;$ (c) the probability density in the $\left|a_{1}\right\rangle$ state in the $\left|S_{0}\right\rangle,\left|T_{0}\right\rangle$ basis, as a function of $B_{1}$. The spectra are obtained using the exact diagonalization method.

\subsection{Haldane Phase in a Chain of Triple Quantum Dots in a Field Effect Transistor}

The first system realizing a synthetic spin one chain [46] is shown in Figure 3a. By patterning the top metallic gate in a typical metal-oxide-semiconductor field-effect transistor (MOSFET) device, one can create local potential minima in the two-dimensional electron gas (2DEG), each of them representing a quantum dot (QD) which can be loaded with a controlled number of electrons [3,7,51-53]. We propose that these dots are arranged in a chain of $N$ triangular molecules [6], as visible in Figure 3a,b.

We describe such a system with an extended Hubbard model $[54,55]$ with Hamiltonian

$$
H_{H}=\sum_{i \sigma} \varepsilon_{i \sigma} c_{i \sigma}^{\dagger} c_{i \sigma}+\sum_{i \neq j, \sigma} t_{i j} c_{i \sigma}^{\dagger} c_{j \sigma}+U \sum_{i} n_{i \uparrow} n_{i \downarrow}+\frac{1}{2} \sum_{i \neq j} V_{i j} n_{i} n_{j}
$$

Here, $c_{i \sigma}\left(c_{i \sigma}^{\dagger}\right)$ is the annihilation (creation) of an electron at dot $i(i=1, \ldots, 3 N), n_{i \sigma}=c_{i \sigma}^{\dagger} c_{i \sigma}$ and $n_{i}=n_{i \uparrow}+n_{i \downarrow}$. The parameter $\varepsilon_{i}$ is the onsite energy, $t_{i j}$ are the hopping integrals, $U$ is the onsite Hubbard interaction and $V_{i j}$ is inter-site density-density Coulomb interaction. We use nonzero $V_{i j}$ and $t_{i j}$ only for nearest-neighboring dots, with $t_{i j}=t, V_{i j}=V$ if they belong to the same molecule and $t_{i j}=t^{\prime}, V_{i j}=V^{\prime}$ if they belong to different molecules (see Figure $3 \mathrm{~b}$ ). The system is filled with four electrons per molecule. If $U, V, V^{\prime} \gg t$, the number of electrons per dot is well defined. Since the "corner" dots have only two neighbors, compared to four neighbors of the dots in the "main chain", they will accumulate more charge. For a range of interaction parameters, the corner dots contain two electrons each, while the main chain has one electron per dot. Note that the dots at the ends of the chain also have only two neighbors. To prevent the accumulation of additional charge on these dots, we adopt the following values of $\varepsilon_{i}: \varepsilon_{i}=V$ at the ends and $\varepsilon_{i}=0$ otherwise. 
(a)

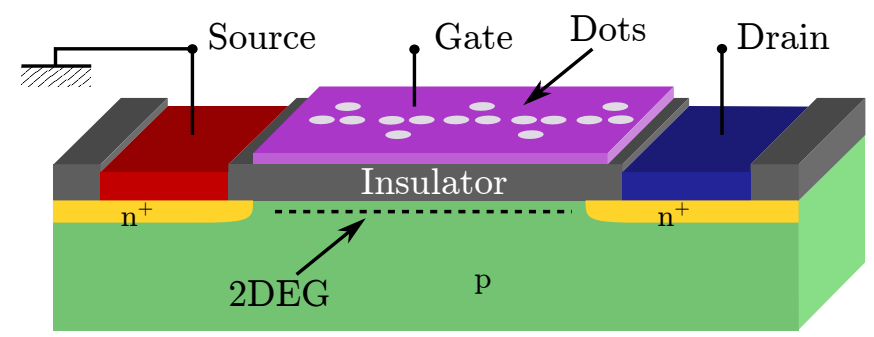

(b)

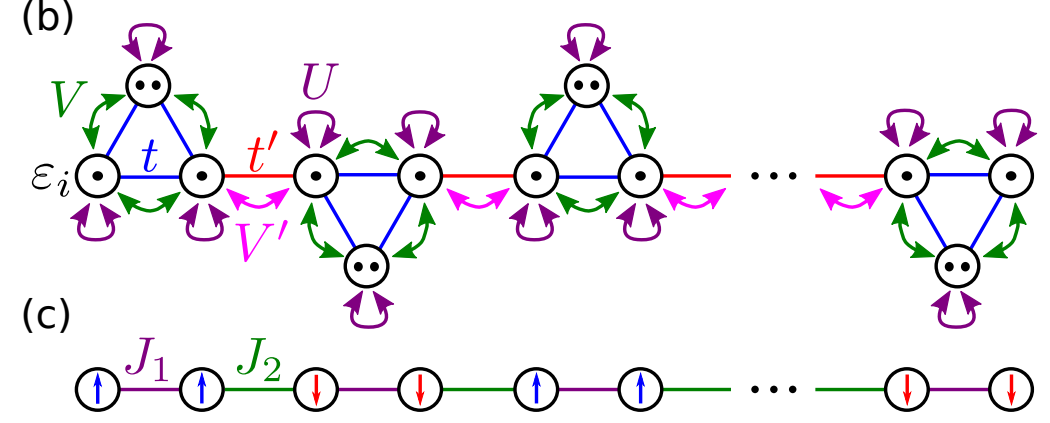

Figure 3. The artificial Haldane chain constructed from triple quantum dot molecules. (a) a schematic view of the system; (b) the parameters of the Hubbard-like model. The circles denote the quantum dots (QDs) and the black dots denote the electrons. The lines and arrows symbolize the single-particle hoppings and interaction terms, respectively; (c) the parameters of the spin-1/2 Heisenberg model.

The low-energy physics of this model can be understood in terms of an effective spin-1/2 chain, which can be obtained via third-order perturbation theory [56]. The model involves only the singly-occupied sites of the "main chain". Electrons occupying them are treated as localized spins-1/2. The effective Hamiltonian is

$$
H_{e f f}=E_{0}+J_{1} \sum_{m=1}^{N} \mathbf{s}_{3 m-2} \cdot \mathbf{s}_{3 m}+J_{2} \sum_{m=1}^{N-1} \mathbf{s}_{3 m} \cdot \mathbf{s}_{3 m+1} .
$$

See also Figure 3c. Here, $\mathbf{s}_{i}$ is the spin-1/2 operator for dot $i$ (with dots numbered from left to right), and

$$
\begin{aligned}
& E_{0}=N U+5 N V+(N+1) V^{\prime}-\frac{2 t^{2} N}{V^{\prime}}-\frac{t^{2} N}{U-V}-\frac{t^{\prime 2}(N-1)}{U-V^{\prime}}-\frac{|t|^{3} N}{V^{\prime 2}} \\
& J_{1}=\frac{4 t^{2}}{U-V}-\frac{4|t|^{3}}{V^{\prime 2}} \\
& J_{2}=\frac{4 t^{\prime 2}}{U-V} .
\end{aligned}
$$

The $J_{2}$ and positive term in $J_{1}$ arise via second-order perturbation theory, as it is usually considered for Hubbard model at half-filling. The negative term in $J_{1}$ arises from third-order processes involving the "corner" dots. By tuning the parameters, it is possible to achieve negative $J_{1}$.

Such an alternating ferromagnetic-antiferromagnetic chain reduces to the spin-1 antiferromagnetic chain with $J=4 J_{2}$ for $J_{1} \gg J_{2}$, when strong ferromagnetic coupling turns pairs of spins into triplets (i.e., spins-1) [57,58]. However, even for finite values of $J_{1} / J_{2}$, the system exhibits the Haldane phase. Decreasing $J_{1} / J_{2}$ increases the gap, yielding a maximal gap equal $J_{2}$ at $J_{1} / J_{2}=0$, in which case the system corresponds to the state of the auxiliary system in the AKLT model, with disconnected singlet dimers and two uncoupled spins at the edges [57].

Based on the first realization of the triple QD molecule [51] and its theoretical charging diagram [54], we choose the example values of parameters $U=2.0, V=0.5, V^{\prime}=0.2, t=-0.05$ and $t^{\prime}=-0.02$, in the units of the effective Rydberg defined by $\mathrm{Ry}^{*}=m^{*} e^{4} /\left(2 \epsilon^{2} \hbar^{2}\right)$, where $m^{*}$ is the 
electron effective mass, $e$ is the electron charge and $\epsilon$ is the dielectric constant. For example, for GaAs, $\mathrm{Ry}^{*}$ is about $6 \mathrm{meV}$.

The validity of the model was confirmed by comparing the exact-diagonalization results in the spin-1/2 model and original Hubbard model, yielding similar results. From perturbation theory, we obtain $J_{1}=-5.83 \times 10^{-3} \mathrm{Ry}^{*}$ and $J_{2}=8.89 \times 10^{-4} \mathrm{Ry}^{*}$. This leads to the Haldane gap $1.07 \mu \mathrm{eV}$, which corresponds to $T=12.4 \mathrm{mK}$. By tuning the ratio $J_{1} / J_{2}$, we can increase it to $T=62 \mathrm{mK}$. The needed magnetic field may be estimated from the condition $g \mu_{B} B_{\mathrm{bg}} / J=0.17$ (see Section 2.2). Using the bulk $g$-factor of GaAs equal 0.41 [59], we obtain $B_{\mathrm{bg}}=0.093 \mathrm{~T}$ for the maximized gap. While this shows that the realization of Haldane phase in a synthetic system is possible, the obtained gap values are rather small. One idea for increasing the gap is to use self-assembled dots, for which the typical values of parameters will be $U=20 \mathrm{meV}, V^{\prime}=10 \mathrm{meV}, t^{\prime}=10 \mathrm{meV}$ [60], for which $J_{2} \sim 40 \mathrm{meV}$ exceeds room temperature. Based on theoretical calculations of Zeeman splitting in self-assembled InAs QDs [61], we estimate that magnetic field $13.6 \mathrm{~T}$ would be necessary to achieve the situation from Section 2.2. Note that the required magnetic field is proportional to $J$ (and hence the Haldane gap), so there is a trade-off between increasing the gap and lowering the field. Nevertheless, we note that the Haldane gap can be tuned, e.g., by controlling the interdot distances, to accomplish a maximum value within the range of achievable magnetic fields.

\subsection{Haldane Phase in a Chain of Semiconductor Quantum Dots in a Nanowire}

To realize a synthetic spin- 1 chain operating at room temperature, we propose a chain of disk-shaped self-assembled QDs (e.g., InAs) embedded in a semiconductor nanowire (InP), as shown in Figure 4a. The construction of one or two such dots was already demonstrated experimentally [62-65]. We describe such a system within an effective mass approximation. The energy spectrum of a single dot is shown in Figure 4b. It resembles a spectrum of a 2D harmonic oscillator, with a nondegenerate $s$-like ground state and a two-fold degenerate $p$ shell, where the states $p_{ \pm}$correspond to angular momentum $L^{z}= \pm 1$. The dots can be loaded with a controlled number of electrons using gates [66,67]. If a dot contains four electrons, two of them fill the $s$ state and have zero total spin, while the exchange interaction aligns ferromagnetically two others filling up the $p$ shell in accordance with the Hund's rule. Thus, they form an effective spin one (see Figure 4c). The antiferromagnetic interaction occurs due to the tunneling between the dots in a nanowire.

(a)

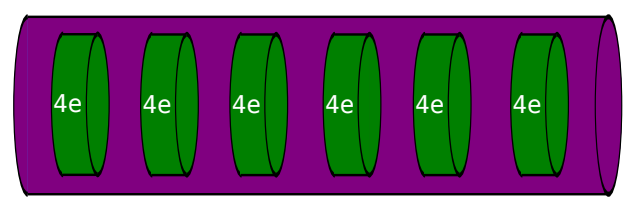

(c)

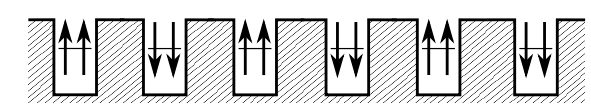

(d)

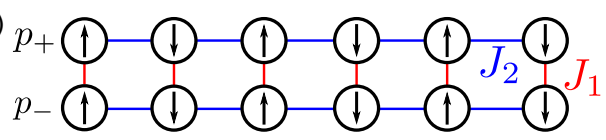

(b)

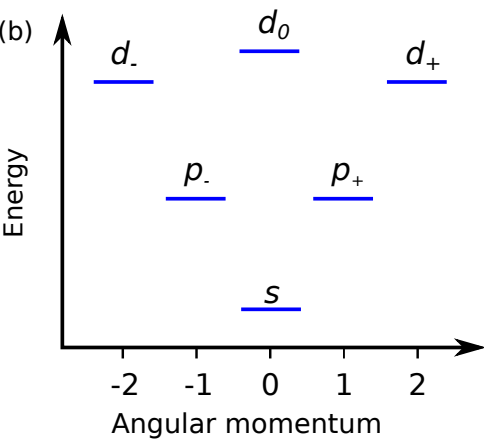

Figure 4. Artificial Haldane chain in a chain of quantum dots embedded in a nanowire. (a) the schematic view of the chain. The green disks are the QDs, the violet cylinder is the nanowire, and " $4 \mathrm{e}$ " denotes filling each dot by four electrons; (b) the low-energy single-particle energy spectrum of a single dot as a function of the angular momentum; (c) the schematic view of the ground state of the whole chain. The two electrons on $p$ orbitals of each dot polarize ferromagnetically, which is signified by parallel arrows. The tunneling between the dots leads to antiferromagnetic polarization of the effective spins-1, which is denoted by antiparallel arrows in neighboring dots; (d) an effective spin ladder, in which every $p$ orbital is treated as localized spin. The coupling is ferromagnetic on the rungs $\left(J_{1}\right)$ and antiferromagnetic on the legs $\left(J_{2}\right)$. 
We can introduce an effective spin ladder by assuming that each $p$ orbital is filled with one electron, and treating it as localized spin-1/2; see Figure 4d. The Hamiltonian is

$$
H_{\text {ladder }}=J_{1} \sum_{i=1}^{N} \mathbf{s}_{i+} \mathbf{s}_{i-}+J_{2} \sum_{i=1}^{N-1}\left(\mathbf{s}_{i+} \mathbf{s}_{(i+1)+}+\mathbf{s}_{i-} \mathbf{s}_{(i+1)-}\right),
$$

where $\mathbf{s}_{i+}, \mathbf{s}_{i-}$ are the spin- $1 / 2$ operators for an electron on $p_{+}$and $p_{-}$orbital of dot $i$, respectively. The ferromagnetic interaction $J_{1}<0$ on the rungs comes from the exchange interaction within each dot. The antiferromagnetic coupling on the legs, $J_{2}=4 t^{2} / U$, can be obtained by describing a single leg within the Hubbard model and considering it within the second-order perturbation theory. Here, $U$ is the onsite Coulomb matrix element, and $t$ is the hopping integral describing the tunneling. If $J_{1} \gg J_{2}$, then this ladder reduces to a spin-1 Heisenberg model with $J=2 J_{2}$. Again, the Haldane phase persists also for finite $J_{1} / J_{2}$ [68]. A simple estimate of the maximum strength of $J$ can be obtained by considering the two-dot case and approximating each dot by a 2D harmonic oscillator. Then, we obtain $U=0.6875 \sqrt{\pi} \sqrt{\omega_{0}} R \mathrm{Ry}^{*}$, where $\omega_{0}$ is the splitting between the shells. The maximum $t$ can be obtained noting that the shells will be distinguishable only when the splitting between the hybridized $p_{ \pm}$orbitals of two dots is less than shell splitting, i.e., maximum $t$ is $t=\omega_{0} / 2$. If we take $4 \mathrm{Ry}^{*}$ as the typical energy spacing, we obtain $J \approx 12 \mathrm{Ry}^{*} \approx 70 \mathrm{meV}$, which is comparable to the room temperature. Again, we can estimate the required magnetic field from $g \mu_{B} B_{\mathrm{bg}} / J=0.17$. The $g$-factor of the cylindrical InAs/InP quantum dots strongly depends on the dot height and diameter [69]. Using $g=6$, being close to the largest values obtained by van Bree et al. [69], we estimate the necessary magnetic field in the case of maximized gap to be about $B_{\mathrm{bg}}=34 \mathrm{~T}$, which is large, thus it may be desirable to consider lower gaps which would require lower fields. Again, the $J$ parameter can be tuned, for example, by controlling interdot distances. We note that an orbital structure similar to Figure $4 \mathrm{~b}$ can be realized in optical lattices, so they can be considered as a potential alternative realization of the above scheme [70].

\subsection{Implementation of the Qubit}

Both approaches result in a qubit which is macroscopic, like superconducting qubits, and semiconductor-based, which means easily integrated with other semiconductor devices and manipulated/addressed optically. The interaction between such qubits needs to be investigated, we note however that coupling of electron-based singlet-triplet qubits was demonstrated already [71].

The topological nature of the Haldane phase would protect the qubits from imperfections such as variation of dot size, shape and separation, which would lead to variation of $J$ in the effective Heisenberg model [72]. If decoherence due to the coupling with nuclear spins turn out to be a problem, one can consider changing the material, e.g., use $\mathrm{Si} / \mathrm{SiGe}$ instead of $\mathrm{InAs} / \mathrm{InP}$ in the case of dots-in-nanowire scenario [73,74]. In addition, we note that, if we do not require the qubits to be macroscopic, one can also think of constructing similar systems on the atomic scale. For example, chains of dangling bonds in silicon can be formed, and precise control of charge in each bond is possible [75].

In addition to the application in the quantum computing, our systems can be useful for fundamental research. In contrast to the current realizations of the Haldane phase, it allows for studying a single chain instead of an ensemble, to tune the model parameters and to use optical methods (instead of, e.g., neutron scattering $[40,41]$ ) to investigate the Haldane phase.

Finally, let us remark that our design is not the only proposal for using the Haldane phase in quantum computing. It has been suggested that each effective edge spin can be used as a separate spin qubit [38]. Another idea is to use the Haldane phase in the measurement-based computation. Because it is based on substantially different principles than the macroscopic singlet-triplet qubit, the discussion of this proposal is beyond the scope of this article. We refer the reader to the original works for more information [36,37]. However, we note that these alternative approaches may also benefit from our construction of synthetic Haldane chains. 


\section{Majorana Qubits}

\subsection{Kitaev Chain}

Now, let us compare our Haldane spin one chain with spin $1 / 2$ quasiparticles at the ends with a Kitaev chain hosting Majorana modes at its edges. The simplest realization would be to take our chain of quantum dots in a nanowire, Figure 4, put it on a $p$-wave superconductor and apply a very large magnetic field along the nanowire axis [76]. The proximity of a nanowire with a chain of quantum dots to a superconductor would result in pairing of spin polarized electrons and fluctuating electron numbers within the dots. The competition of dot-dot tunneling, interdot pairing and variable electron numbers are captured within a model proposed by Kitaev [26],

$$
H=t \sum_{n=1}^{N-1}\left(c_{n+1}^{\dagger} c_{n}+\text { H.c. }\right)+\Delta \sum_{n=1}^{N-1}\left(c_{n+1}^{\dagger} c_{n}^{\dagger}+\text { H.c. }\right)-\mu \sum_{n=1}^{N} c_{n}^{\dagger} c_{n} .
$$

Here, $n$ numbers the s-orbitals in a 1D chain of quantum dots of length $N, c_{n}^{\dagger}\left(c_{n}\right)$ is a creation (annihilation) operator of a spinless fermion at site $n, t$ is a hopping integral, $\Delta$ is the SC pairing energy for electrons on neighboring sites, and $\mu$ is the chemical potential. The Hamiltonian does not conserve the number of electrons as electron pairs are fluctuating between the nanowire and a superconductor. Thus, single-particle energies in this model are the energies of Bogoliubov quasiparticles. Here, we will focus on the emergence of Majorana zero energy modes, while its physical realization will be briefly discussed later.

We start by rewriting Kitaev's Hamiltonian in terms of Majorana operators. The Majorana fermions are particles which are their own antiparticles, proposed by Ettore Majorana as real solutions of the Dirac equation [77]. Following this idea, fermionic operators on site $n$ are represented by a superposition of two real Majorana operators $\gamma_{n, 1}$ and $\gamma_{n, 2}$,

$$
c_{n}^{\dagger}=\frac{1}{2}\left(\gamma_{n, 1}-i \gamma_{n, 2}\right), \quad c_{i}=\frac{1}{2}\left(\gamma_{n, 1}+i \gamma_{n, 2}\right)
$$

The Majorana operators satisfy

$$
\gamma_{n, \sigma}^{\dagger}=\gamma_{n, \sigma}, \quad \gamma_{m, \sigma_{1}} \gamma_{n, \sigma_{2}}+\gamma_{n, \sigma_{2}} \gamma_{m, \sigma_{1}}=2 \delta_{n m} \delta_{\sigma_{1}, \sigma_{2}}, \quad \gamma_{n, \sigma}^{2}=1,
$$

where $\sigma, \sigma_{1}, \sigma_{2}=1,2$.

The Hamiltonian, Equation (9), can be now expressed in Majorana operators:

$$
H=\frac{i}{2}(t-\Delta) \sum_{j=1}^{N-1} \gamma_{j+1,1} \gamma_{j, 2}-\frac{i}{2}(t+\Delta) \sum_{j=1}^{N-1} \gamma_{j+1,2} \gamma_{j, 1}-\frac{\mu}{2} \sum_{j=1}^{N}\left(1+i \gamma_{j, 1} \gamma_{j, 2}\right) .
$$

We now show how the Hamiltonian, Equation (12), can be diagonalized for a special case $\Delta=t$ and $\mu=0$. We start by pairing Majorana operator $\gamma_{n, 1}$ on site $n$ with other Majorana operator $\gamma_{n+1,2}$ on neighboring site, $n+1$, through a new fermionic pair operator $a_{n}$ :

$$
a_{n}^{\dagger}=\left(\gamma_{n, 1}-i \gamma_{n+1,2}\right) / 2 .
$$

The set of new pair operators allows us to diagonalize the Hamiltonian for $\Delta=t$ and $\mu=0$,

$$
H=-i t \sum_{j=1}^{N-1} \gamma_{j+1,2} \gamma_{j, 1}=t \sum_{n=1}^{N-1}\left(2 a_{n}^{\dagger} a_{n}-1\right),
$$

Now, the Hamiltonian pairs Majorana operators corresponding to different sites as illustrated in Figure $5 a$, resulting in states of Bogoliubov quasiparticles with energies $\pm t= \pm \Delta$. However, there 
are two unpaired Majorana operators at the edges, $\gamma_{1,2}$, and $\gamma_{N, 1}$. Since they do not appear in the Hamiltonian, they correspond to zero-energy eigenstates known as Majorana zero modes (MZMs). Note the similarity to the Haldane chain-again, we expressed each physical operator (fermionic creation or annihilation operator) with two auxiliary ones (Majorana operators), and paired them in such a way that two auxiliary operators at the edges remain free.

Just like the Haldane edge spins, the existence of the Majorana zero modes is not limited to certain parameter values. They are topologically protected, i.e., they are stable with respect to continuous transformation of the Hamiltonian as long as the energy gap does not close, that is, as long as $|\mu|<2|t|$, $\Delta \neq 0$ (although in general their structure is more complicated than at $\Delta=t, \mu=0$ in the sense that they are not perfectly localized at one site, but are exponentially localized at the edges). We will call the region with MZM "topological" and the region without MZMs "trivial". The existence of MZMs is tied to a $\mathbb{Z}_{2}$ topological invariant, characterizing the band structure of an infinite chain [15]. In contrast to the Haldane phase, which is protected by certain symmetries, the MZMs are stable to any perturbation. This is because connecting a topological and trivial Kitaev chain adiabatically would mean violating the fermion parity symmetry.


(c)

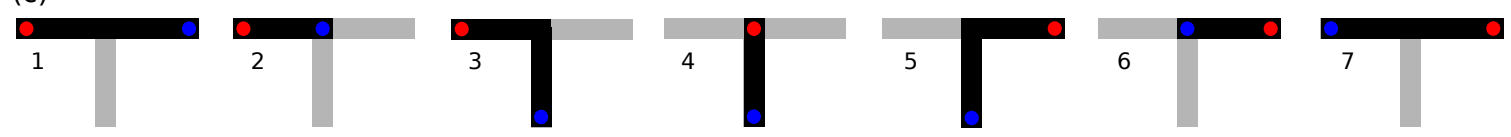

Figure 5. The qubits based on Majorana chain. (a) a schematic view of the single-particle eigenstates of Kitaev model in the special case of $\Delta=t, \mu=0$. The blue rectangles represent the sites, described with fermionic creation operators $c_{i}^{\dagger}$, the black circles represent Majorana operators, and the red ellipses denote pairing two Majorana operators into a pair operator $a_{i}^{\dagger}$, being a fermionic creation operator; (b) four Majorana zero modes (MZMs) in a network of nanowires. The gray and black denote the trivial and topological regions of the wires, respectively. The blue and red dots represent the MZMs. (c) braiding of two Majorana modes. The meaning of colors is the same as in (b). Although the MZMs are indistinguishable, we have labeled them with two different colors for clarity.

\subsection{Non-Abelian Statistics and Topological Quantum Computations}

The potential of the MZMs for the quantum computing lies in their non-Abelian statistics [78,79]. The braiding operations, i.e., encircling one MZM by another one or exchanging their position, result in mixing of several degenerate ground states [18]. This degeneracy can be understood by noting that a pair of edge MZMs $\gamma_{1,2}, \gamma_{N, 1}$ can be expressed as a fermionic state $a_{0}^{\dagger}=\left(\gamma_{1,2}-i \gamma_{N, 1}\right) / 2$, which can be either occupied or filled, and both options correspond to zero energy. Thus, $2 N_{c}$ MZMs (appearing in $N_{c}$ Kitaev chains) lead to the presence of $2^{N_{c}}$ degenerate ground states. These states can be used to collectively represent a set of qubits, and quantum gates can be encoded in the braiding operations [15,29-31]. Since the effect or the braiding does not depend on the details of the trajectory, only on its topology (e.g., on the fact if a given particle encircled another particle or not), it would be protected from decoherence. This is called topological quantum computing.

Naively, a single qubit could be implemented using a single chain: the $a_{0}^{\dagger}$ fermionic state can be either empty or filled, which we could choose as two qubit states. However, both the Hamiltonian and the braiding operations conserve fermionic parity, so one cannot achieve a superposition of the two qubit states. To overcome this problem, one can encode a qubit in two chains, in analogy to the singlet triplet qubit discussed previously. Let us consider four Majorana modes: $\gamma_{1}, \gamma_{2}$ being the end states of first chain and $\gamma_{3}, \gamma_{4}$ being the end states of second chain. We can use them to form two fermionic 
states $\tilde{a}_{1}^{+}=\left(\gamma_{1}-i \gamma_{2}\right) / 2$, and $\tilde{a}_{2}^{+}=\left(\gamma_{3}-i \gamma_{4}\right) / 2$. We consider a qubit encoded in two states of the even-parity subspace, $|00\rangle$ and $|11\rangle=\tilde{a}_{1}^{+} \tilde{a}_{2}^{\dagger}|00\rangle[29-31,80]$.

The effect of exchanging MZMs $i$ and $j$ is described by an unitary operator $[78,79]$

$$
\exp \left( \pm \frac{\pi}{4} \gamma_{i} \gamma_{j}\right)=\frac{1}{\sqrt{2}}\left(1 \pm \gamma_{i} \gamma_{j}\right)
$$

with \pm depending on the direction of the exchange. Equation (15) describes the statistics of the class of anyons called Ising anyons. As an example of a quantum gate encoded using braiding, let us consider the effect of exchanging $\gamma_{2}$ and $\gamma_{3}$ twice in the direction corresponding to " + " in Equation (15),

$$
\frac{1}{\sqrt{2}}\left(1+\gamma_{2} \gamma_{3}\right)^{2}=\gamma_{2} \gamma_{3}=i\left(\tilde{a}_{1}^{\dagger} \tilde{a}_{2}^{+}+\tilde{a}_{2} \tilde{a}_{1}\right)
$$

where we considered only the terms acting within the qubit Hibert space $\{|00\rangle,|11\rangle\}$. Such an operation exchanges the two qubit states and implements a $i \sigma_{x}$ gate. In similar way, one can encode e.g., a $\pi / 2$ phase gate and the Hadamard gate [29-31].

Representing several qubits can be achieved in at least two ways [80]. A "sparse" encoding uses two chains per qubit ( $N_{c}=2 N_{\mathrm{QB}}, N_{\mathrm{QB}}$ being the number of qubits), each one created as described above. On the other hand, the Hilbert space of $N_{c}$ chains contains $2^{N_{c}-1}$ states in each parity sector. Thus, a "dense encoding" is possible with only $N_{c}=N_{\mathrm{QB}}+1$ chains representing $N_{\mathrm{QB}}$ qubits collectively. In the dense encoding, two-qubit gates can be encoded solely by braiding [30]. In the sparse encoding, the entanglement between the qubits cannot be achieved using braiding only [31], and one should complement the braiding with measurements [80].

Unfortunately, for Ising anyons, the gate set constructed using braiding and measurements does not allow for universal quantum computations [31]. It should be complemented with one operation which is not protected topologically, a $\pi / 8$ phase gate. A high-fidelity implementation of such a gate relies on a procedure called "magic state distillation" [31,81]. In the case of topological qubits, such a procedure can be performed on less qubits than in conventional quantum circuits, which is an advantage of the topological setup [80].

How can the braiding operations be performed if the system is one-dimensional, and the MZMs are glued to the ends of the chain? If the chemical potential is not a constant, but varies along the chain, the system can be divided into topological and trivial parts, with MZMs appearing on the endpoints of each topological region. We can consider a system which is not a single chain, but a network of chains (see Figure $5 b$ ). By controlling the chemical potential at each point of the network, the topological parts can be shifted, and the MZMs can be moved and braided [79]. For example, two MZMs can be exchanged in a T-shaped junction, as shown in Figure 5c. Other braiding schemes also exist [82,83].

\subsection{Physical Realization}

Let us now briefly discuss how the Kitaev model can be realized in practice. For the existence of unpaired Majorana modes, it is essential that the particles are spinless. If we simply take two copies of Kitaev model corresponding to two spins, we will obtain two Majorana modes at each end, i.e., they will be paired and therefore not protected topologically. Spin degeneracy can be lifted e.g., in a semiconductor nanowire or a chain of quantum dots by a combination of spin-orbit coupling and magnetic field. The superconductivity can be induced by proximity effect, i.e., by placing the nanowire close to a superconductor and allowing the Cooper pairs to tunnel into the nanowire. The specific kind of superconductivity in the Kitaev model ( $p$-wave, pairing spinless particles or particles with the same spin) is significantly different than the $s$-wave pairing in ordinary superconductors where particles with opposite spins create singlet Cooper pairs. Nevertheless, the combination of the spin-orbit coupling, magnetic field and pairing induced by the proximity of an $s$-wave superconductor results 
in a system which can be mapped to Kitaev model $[27,28,76]$. Another proposal for creating MZMs involves depositing a magnetic atomic chain on the top of the superconductor $[84,85]$.

Systems like this were already constructed experimentally, and the existence of edge states has been shown [86]. However, such states are not necessarily the MZMs; they may be, e.g., Andreev bound states. Recently, the experiments have shown edge states exhibiting local conductance agreeing with the theoretical result for MZMs [33], which rules out some alternative scenarios. Another observed signature of Majorana zero modes is the fractional Josephson effect $[87,88]$. Nevertheless, a definite proof would require the demonstration of braiding, which is not achieved yet. For the discussion of other signatures of MZMs, the recent progress and alternative explanations of experimental results, we refer the reader to Lutchyn et al. [89] and references therein.

There is also ongoing theoretical work on several aspects of the MZMs, to allow a good description of the experiments. For example, in real systems, the electrons inside the nanowire will interact, which is not included in the Kitaev model (apart from the mean-field pairing terms which are related to the creation of Cooper pairs in the superconductor, not the interaction within the nanowire). This may affect the formation of MZMs $[90,91]$. In addition, there are proposals to realize the Majorana modes in number-conserving models, which may make it easier to create them with cold atoms in optical lattices [92,93].

Finally, let us note that, in general, the existence of Majorana zero modes is not limited to the Kitaev model. They can exist in two-dimensional topological superconductors [94,95], as well as the Moore-Read quantum Hall state [24]. This subject is beyond the scope of this article, hence we refer the Reader to Das Sarma et al. [80], Beenakker et al. [96] and Alicea et al. [97] and references therein.

\section{Conclusions}

In this work, we have reviewed our work on constructing quantum bits with macroscopic, topologically protected, states in semiconductor devices. We discussed two approaches using the edge states of one-dimensional topological phases, the synthetic Haldane and Kitaev chains of quantum dots. In both cases, the topological protection is used to make the qubit robust against perturbations. The two phases can be understood based on a similar scheme of expressing each physical site as two auxiliary sites and coupling the nearest-neighboring auxiliary sites in such a way that two of them remain unpaired at the ends. This is related to the fact that the topological phases in 1D can be constructed as matrix product states [98,99]. In fact, different 1D topological phases, including the Haldane phase, may be understood as several coupled Majorana chains [100,101].

On the other hand, the two qubit implementations are radically different. In the case of the Haldane phase, the edge states are manipulated in a way similar to ordinary spins, while, in the case of a Majorana qubit, the manipulations correspond to physically moving the endpoints of chains to braid them. Both methods have their strengths and weaknesses. The Haldane phase qubit is based on a phase of matter whose existence was already confirmed, in contrast to the MZMs. Haldane qubits would be fully semiconductor-based, and thus can be easily integrated with other semiconductor devices, while the Majorana qubits need the proximity of a superconductor. On the other hand, the topological protection in the case of MZMs is stronger, as it does not rely on symmetry. Finally, the theory of Haldane qubits is not fully developed; further studies are needed to describe initialization, readout, realization of various gates and coupling with other qubits, as well as to perform detailed analysis of decoherence mechanisms. On the other hand, the designs of quantum circuits based on Majorana qubits do exist $[79,83,89]$.

Clearly, much work is still needed to advance the field of quantum computation with macroscopic, topologically protected, states in semiconductor devices.

Author Contributions: Conceptualization, P.H.; Methodology, P.H.; Software, B.J.; Validation, P.H. and B.J.; Formal Analysis, P.H. and B.J.; Investigation, P.H. and B.J.; Resources, P.H. and B.J; Data Curation, B.J.; Writing-Original Draft Preparation, B.J.; Writing-Review and Editing, P.H. and B.J.; Visualization, B.J.; Supervision, P.H.; Project Administration, P.H.; Funding Acquisition, P.H. and B.J. 
Funding: B.J. acknowledges funding from the National Science Centre (NCN), Poland, Grant Maestro No. 2014/14/A/ST3/00654. P.H. and B.J. acknowledge support by NSERC and the uOttawa Chair in the Quantum Theory of Materials, Nanostructures and Devices.

Conflicts of Interest: The authors declare no conflict of interest.

\section{Abbreviations}

The following abbreviations are used in this manuscript:

AKLT Affleck, Kennedy, Lieb, Tasaki

QD quantum dot

MZM Majorana zero mode

SC superconductor/superconducting

\section{References}

1. Brum, J.A.; Hawrylak, P. Coupled quantum dots as quantum exclusive-OR gate. Superlattices Microstruct. 1997, 22, 431-436. [CrossRef]

2. Loss, D.; DiVincenzo, D.P. Quantum computation with quantum dots. Phys. Rev. A 1998, 57, $120-126$. [CrossRef]

3. Ciorga, M.; Sachrajda, A.S.; Hawrylak, P.; Gould, C.; Zawadzki, P.; Jullian, S.; Feng, Y.; Wasilewski, Z. Addition spectrum of a lateral dot from Coulomb and spin-blockade spectroscopy. Phys. Rev. B 2000, 61, R16315-R16318. [CrossRef]

4. Watson, T.F.; Philips, S.G.J.; Kawakami, E.; Ward, D.R.; Scarlino, P.; Veldhorst, M.; Savage, D.E.; Lagally, M.G.; Friesen, M.; Coppersmith, S.N.; et al. A programmable two-qubit quantum processor in silicon. Nature 2018, 555, 633-637. [CrossRef] [PubMed]

5. Korkusinski, M.; Hawrylak, P. Coded qubits based on electron spin. In Semiconductor Quantum Bits; Benson, O., Henneberger, F., Eds.; Pan Stanford: Singapore, 2009.

6. Hsieh, C.Y.; Shim, Y.P.; Korkusinski, M.; Hawrylak, P. Physics of lateral triple quantum-dot molecules with controlled electron numbers. Rep. Prog. Phys. 2012, 75, 114501. [CrossRef] [PubMed]

7. Petta, J.R.; Johnson, A.C.; Taylor, J.M.; Laird, E.A.; Yacoby, A.; Lukin, M.D.; Marcus, C.M.; Hanson, M.P.; Gossard, A.C. Coherent Manipulation of Coupled Electron Spins in Semiconductor Quantum Dots. Science 2005, 309, 2180-2184. [CrossRef] [PubMed]

8. Dial, O.E.; Shulman, M.D.; Harvey, S.P.; Bluhm, H.; Umansky, V.; Yacoby, A. Charge Noise Spectroscopy Using Coherent Exchange Oscillations in a Singlet-Triplet Qubit. Phys. Rev. Lett. 2013, 110, 146804. [CrossRef] [PubMed]

9. Nizovtsev, A.P.; Kilin, S.Y.; Jelezko, F.; Gaebal, T.; Popa, I.; Gruber, A.; Wrachtrup, J. A quantum computer based on NV centers in diamond: Optically detected nutations of single electron and nuclear spins. Opt. Spectrosc. 2005, 99, 233-244. [CrossRef]

10. Neumann, P.; Mizuochi, N.; Rempp, F.; Hemmer, P.; Watanabe, H.; Yamasaki, S.; Jacques, V.; Gaebel, T.; Jelezko, F.; Wrachtrup, J. Multipartite Entanglement Among Single Spins in Diamond. Science 2008, 320, 1326. [CrossRef]

11. Saeedi, K.; Simmons, S.; Salvail, J.Z.; Dluhy, P.; Riemann, H.; Abrosimov, N.V.; Becker, P.; Pohl, H.J.; Morton, J.J.L.; Thewalt, M.L.W. Room-Temperature Quantum Bit Storage Exceeding 39 Minutes Using Ionized Donors in Silicon-28. Science 2013, 342, 830-833. [CrossRef]

12. Pla, J.J.; Tan, K.Y.; Dehollain, J.P.; Lim, W.H.; Morton, J.J.L.; Zwanenburg, F.A.; Jamieson, D.N.; Dzurak, A.S.; Morello, A. High-fidelity readout and control of a nuclear spin qubit in silicon. Nature 2013, 496, 334-338. [CrossRef] [PubMed]

13. Barends, R.; Shabani, A.; Lamata, L.; Kelly, J.; Mezzacapo, A.; Heras, U.L.; Babbush, R.; Fowler, A.G.; Campbell, B.; Chen, Y.; et al. Digitized adiabatic quantum computing with a superconducting circuit. Nature 2016, 534, 222-226. [CrossRef] [PubMed]

14. Johnson, M.W.; Amin, M.H.S.; Gildert, S.; Lanting, T.; Hamze, F.; Dickson, N.; Harris, R.; Berkley, A.J.; Johansson, J.; Bunyk, P.; et al. Quantum annealing with manufactured spins. Nature 2011, 473, 194-198. [CrossRef] [PubMed]

15. Kitaev, A.Y. Fault-tolerant quantum computation by anyons. Ann. Phys. 2003, 303, 2-30. [CrossRef] 
16. Leinaas, J.M.; Myrheim, J. On the theory of identical particles. Il Nuovo Cimento B 1977, 37, 1-23. [CrossRef]

17. Wilczek, F. Quantum mechanics of fractional-spin particles. Phys. Rev. Lett. 1982, 49, 957-959. [CrossRef]

18. Fröhlich, J.; Gabbiani, F. Braid statistics in local quantum theory. Rev. Math. Phys. 1990, 2, 251-353. [CrossRef]

19. Wen, X.G. Colloquium: Zoo of quantum-topological phases of matter. Rev. Mod. Phys. 2017, 89, 041004. [CrossRef]

20. Haldane, F.D.M. Nobel Lecture: Topological quantum matter. Rev. Mod. Phys. 2017, 89, 040502. [CrossRef]

21. Qi, X.L.; Zhang, S.C. Topological insulators and superconductors. Rev. Mod. Phys. 2011, 83, $1057-1110$. [CrossRef]

22. Landau, L.D. The theory of phase transitions I. Zhurnal Eksperimental'noi i Teoreticheskoi Fiziki 1937, 7, 19-32. [CrossRef]

23. Landau, L.D. The theory of phase transitions II. Zhurnal Eksperimental'noi i Teoreticheskoi Fiziki 1937, 7, 627.

24. Moore, G.; Read, N. Nonabelions in the fractional quantum Hall effect. Nucl. Phys. B 1991, 360, $362-396$. [CrossRef]

25. Read, N.; Rezayi, E. Beyond paired quantum Hall states: Parafermions and incompressible states in the first excited Landau level. Phys. Rev. B 1999, 59, 8084-8092. [CrossRef]

26. Kitaev, A.Y. Unpaired Majorana fermions in quantum wires. Physics-Uspekhi 2001, 44, 131. [CrossRef]

27. Lutchyn, R.M.; Sau, J.D.; Das Sarma, S. Majorana Fermions and a Topological Phase Transition in SemiconductorSuperconductor Heterostructures. Phys. Rev. Lett. 2010, 105, 077001. [CrossRef]

28. Oreg, Y.; Refael, G.; von Oppen, F. Helical Liquids and Majorana Bound States in Quantum Wires. Phys. Rev. Lett. 2010, 105, 177002. [CrossRef]

29. Zhang, C.; Tewari, S.; Das Sarma, S. Bell's Inequality and Universal Quantum Gates in a Cold-Atom Chiral Fermionic p-Wave Superfluid. Phys. Rev. Lett. 2007, 99, 220502. [CrossRef]

30. Georgiev, L.S. Topologically protected gates for quantum computation with non-Abelian anyons in the Pfaffian quantum Hall state. Phys. Rev. B 2006, 74, 235112. [CrossRef]

31. Bravyi, S. Universal quantum computation with the $v=5 / 2$ fractional quantum Hall state. Phys. Rev. A 2006, 73, 042313. [CrossRef]

32. Camino, F.E.; Zhou, W.; Goldman, V.J. Realization of a Laughlin quasiparticle interferometer: Observation of fractional statistics. Phys. Rev. B 2005, 72, 075342. [CrossRef]

33. Ferreira, G.J.; Loss, D. Magnetically Defined Qubits on 3D Topological Insulators. Phys. Rev. Lett. 2013, 111, 106802. [CrossRef] [PubMed]

34. Michetti, P.; Recher, P. Bound states and persistent currents in topological insulator rings. Phys. Rev. B 2011, 83, 125420. [CrossRef]

35. Xu, H.; Huang, L.; Lai, Y.C.; Grebogi, C. Superpersistent currents and whispering gallery modes in relativistic quantum chaotic systems. Sci. Rep. 2015, 5, 8963. [CrossRef] [PubMed]

36. Miyake, A. Quantum computation on the edge of a symmetry-protected topological order. Phys. Rev. Lett. 2010, 105, 040501. [CrossRef]

37. Else, D.V.; Schwarz, I.; Bartlett, S.D.; Doherty, A.C. Symmetry-protected phases for measurement-based quantum computation. Phys. Rev. Lett. 2012, 108, 240505. [CrossRef] [PubMed]

38. Katsura, H.; Hirano, T.; Hatsugai, Y. Exact analysis of entanglement in gapped quantum spin chains. Phys. Rev. B 2007, 76, 012401. [CrossRef]

39. Renard, J.P.; Verdaguer, M.; Regnault, L.P.; Erkelens, W.A.C.; Rossat-Mignod, J.; Stirling, W.G. Presumption for a quantum energy gap in the quasi-one-dimensional $S=1$ Heisenberg antiferromagnet $\mathrm{Ni}\left(\mathrm{C}_{2} \mathrm{H}_{8} \mathrm{~N}_{2}\right)_{2} \mathrm{NO}_{2}\left(\mathrm{ClO}_{4}\right)$. Europhys. Lett. 1987, 3, 945. [CrossRef]

40. Buyers, W.J.L.; Morra, R.M.; Armstrong, R.L.; Hogan, M.J.; Gerlach, P.; Hirakawa, K. Experimental evidence for the Haldane gap in a spin-1 nearly isotropic, antiferromagnetic chain. Phys. Rev. Lett. 1986, 56, 371-374. [CrossRef]

41. Steiner, M.; Kakurai, K.; Kjems, J.K.; Petitgrand, D.; Pynn, R. Inelastic neutron scattering studies on 1D near-Heisenberg antiferromagnets: A test of the Haldane conjecture. J. Appl. Phys. 1987, 61, 3953-3955. [CrossRef]

42. Haldane, F.D.M. Nonlinear field theory of large-spin Heisenberg antiferromagnets: Semiclassically quantized solitons of the one-dimensional easy-axis Néel state. Phys. Rev. Lett. 1983, 50, 1153-1156. [CrossRef]

43. Haldane, F.D.M. Continuum dynamics of the 1-D Heisenberg antiferromagnet: identification with the $\mathrm{O}$ (3) nonlinear sigma model. Phys. Lett. A 1983, 93, 464-468. [CrossRef] 
44. Affleck, I.; Haldane, F.D.M. Critical theory of quantum spin chains. Phys. Rev. B 1987, 36, 5291. [CrossRef]

45. Jaworowski, B.; Rogers, N.; Grabowski, M.; Hawrylak, P. Macroscopic Singlet-Triplet Qubit in Synthetic Spin-One Chain in Semiconductor Nanowires. Sci. Rep. 2017, 7, 5529. [CrossRef] [PubMed]

46. Shim, Y.P.; Sharma, A.; Hsieh, C.Y.; Hawrylak, P. Artificial Haldane gap material on a semiconductor chip. Solid State Commun. 2010, 150, 2065-2068. [CrossRef]

47. White, S.R. Density matrix formulation for quantum renormalization groups. Phys. Rev. Lett. 1992, 69, 2863-2866. [CrossRef] [PubMed]

48. Affleck, I.; Kennedy, T.; Lieb, E.H.; Tasaki, H. Rigorous results on valence-bond ground states in antiferromagnets. Phys. Rev. Lett. 1987, 59, 799-802. [CrossRef]

49. Gu, Z.C.; Wen, X.G. Tensor-entanglement-filtering renormalization approach and symmetry-protected topological order. Phys. Rev. B 2009, 80, 155131. [CrossRef]

50. Pollmann, F.; Berg, E.; Turner, A.M.; Oshikawa, M. Symmetry protection of topological phases in one-dimensional quantum spin systems. Phys. Rev. B 2012, 85, 075125. [CrossRef]

51. Gaudreau, L.; Studenikin, S.A.; Sachrajda, A.S.; Zawadzki, P.; Kam, A.; Lapointe, J.; Korkusinski, M.; Hawrylak, P. Stability Diagram of a Few-Electron Triple Dot. Phys. Rev. Lett. 2006, 97, 036807. [CrossRef]

52. Koppens, F.H.L.; Buizert, C.; Tielrooij, K.J.; Vink, I.T.; Nowack, K.C.; Meunier, T.; Kouwenhoven, L.P.; Vandersypen, L.M.K. Driven coherent oscillations of a single electron spin in a quantum dot. Nature 2006, 442, 766-771. [CrossRef] [PubMed]

53. Gaudreau, L.; Kam, A.; Granger, G.; Studenikin, S.A.; Zawadzki, P.; Sachrajda, A.S. A tunable few electron triple quantum dot. Appl. Phys. Lett. 2009, 95, 193101. [CrossRef]

54. Korkusinski, M.; Gimenez, I.P.; Hawrylak, P.; Gaudreau, L.; Studenikin, S.A.; Sachrajda, A.S. Topological Hunds rules and the electronic properties of a triple lateral quantum dot molecule. Phys. Rev. B 2007, 75, 115301. [CrossRef]

55. Delgado, F.; Shim, Y.P.; Korkusinski, M.; Hawrylak, P. Theory of spin, electronic, and transport properties of the lateral triple quantum dot molecule in a magnetic field. Phys. Rev. B 2007, 76, 115332. [CrossRef]

56. Shim, Y.P.; Hawrylak, P. Gate-controlled spin-spin interactions in lateral quantum dot molecules. Phys. Rev. B 2008, 78, 165317. [CrossRef]

57. Hida, K. Crossover between the Haldane-gap phase and the dimer phase in the spin-1/2 alternating Heisenberg chain. Phys. Rev. B 1992, 45, 2207-2212. [CrossRef]

58. Hung, H.H.; Gong, C.D. Numerical evidence of a spin- $1 / 2$ chain approaching a spin-1 chain. Phys. Rev. B 2005, 71, 054413. [CrossRef]

59. Fernandes da Silva, E. GaAs: Effective Landé g-factors. In New Data and Updates for I-VII, III-V, III-VI and IV-VI Compounds; Roessler, U., Ed.; Springer: Berlin/Heidelberg, Germany, 2009; pp. 214-215. [CrossRef]

60. Reimer, M.E.; McKinnon, W.R.; Lapointe, J.; Dalacu, D.; Poole, P.J.; Aers, G.C.; Kim, D.; Korkusiński, M.; Hawrylak, P.; Williams, R.L. Towards scalable gated quantum dots for quantum information applications. Physica E 2008, 40, 1790-1793. [CrossRef]

61. Sheng, W.; Hawrylak, P. Spin polarization in self-assembled quantum dots. Phys. Rev. B 2006, 73, 125331. [CrossRef]

62. Dalacu, D.; Mnaymneh, K.; Wu, X.; Lapointe, J.; Aers, G.C.; Poole, P.J.; Williams, R.L. Selective-area vapor-liquid-solid growth of tunable InAsP quantum dots in nanowires. Appl. Phys. Lett. 2011, 98, 251101. [CrossRef]

63. Wallentin, J.; Anttu, N.; Asoli, D.; Huffman, M.; Åberg, I.; Magnusson, M.H.; Siefer, G.; Fuss-Kailuweit, P.; Dimroth, F.; Witzigmann, B.; et al. InP Nanowire Array Solar Cells Achieving 13.8\% Efficiency by Exceeding the Ray Optics Limit. Science 2013, 339, 1057-1060. [CrossRef] [PubMed]

64. Haffouz, S.; Zeuner, K.D.; Dalacu, D.; Poole, P.J.; Lapointe, J.; Poitras, D.; Mnaymneh, K.; Wu, X.; Couillard, M.; Korkusinski, M.; et al. Bright single InAsP quantum dots at telecom wavelengths in position-controlled InP nanowires: The role of the photonic waveguide. Nano Lett. 2018, 18, 3047-3052. [CrossRef] [PubMed]

65. Dalacu, D.; Mnaymneh, K.; Lapointe, J.; Wu, X.; Poole, P.J.; Bulgarini, G.; Zwiller, V.; Reimer, M.E. Ultraclean Emission from InAsP Quantum Dots in Defect-Free Wurtzite InP Nanowires. Nano Lett. 2012, 12, 5919-5923. [CrossRef] [PubMed]

66. Kouwen, M.P.V.; Reimer, M.E.; Hidma, A.W.; van Weert, M.H.M.; Algra, R.E.; Bakkers, E.P.A.M.; Kouwenhoven, L.P.; Zwiller, V. Single Electron Charging in Optically Active Nanowire Quantum Dots. Nano Lett. 2010, 10, 1817-1822. [CrossRef] 
67. Van Weert, M.H.M.; den Heijer, M.; van Kouwen, M.P.; Algra, R.E.; Bakkers, E.P.A.M.; Kouwenhoven, L.P.; Zwiller, V. Surround-gated vertical nanowire quantum dots. Appl. Phys. Lett. 2010, 96, 233112. [CrossRef]

68. Hida, K. Haldane gap in the spin-1/2 double chain Heisenberg antiferromagnet. J. Magn. Magn. Mater. 1992, 104-107, 783-784. [CrossRef]

69. Van Bree, J.; Silov, A.Y.; Koenraad, P.M.; Flatté, M.E.; Pryor, C.E. $g$ factors and diamagnetic coefficients of electrons, holes, and excitons in InAs/InP quantum dots. Phys. Rev. B 2012, 85, 165323. [CrossRef]

70. Nonne, H.; Moliner, M.; Capponi, S.; Lecheminant, P.; Totsuka, K. Symmetry-protected topological phases of alkaline-earth cold fermionic atoms in one dimension. Europhys. Lett. 2013, 102, 37008. [CrossRef]

71. Shulman, M.D.; Dial, O.E.; Harvey, S.P.; Bluhm, H.; Umansky, V.; Yacoby, A. Demonstration of Entanglement of Electrostatically Coupled Singlet-Triplet Qubits. Science 2012, 336, 202. [CrossRef]

72. Hyman, R.A.; Yang, K.; Bhatt, R.N.; Girvin, S.M. Random Bonds and Topological Stability in Gapped Quantum Spin Chains. Phys. Rev. Lett. 1996, 76, 839-842. [CrossRef]

73. Maune, B.M.; Borselli, M.G.; Huang, B.; Ladd, T.D.; Deelman, P.W.; Holabird, K.S.; Kiselev, A.A.; Alvarado-Rodriguez, I.; Ross, R.S.; Schmitz, A.E.; et al. Coherent singlet-triplet oscillations in a silicon-based double quantum dot. Nature 2012, 481, 344-347. [CrossRef]

74. Hu, Y.; Churchill, H.O.H.; Reilly, D.J.; Xiang, J.; Lieber, C.M.; Marcus, C.M. A Ge/Si heterostructure nanowire-based double quantum dot with integrated charge sensor. Nat. Nanotechnol. 2007, 2, $622-625$. [CrossRef] [PubMed]

75. Rashidi, M.; Vine, W.; Dienel, T.; Livadaru, L.; Retallick, J.; Huff, T.; Walus, K.; Wolkow, R.A. Initiating and Monitoring the Evolution of Single Electrons Within Atom-Defined Structures. Phys. Rev. Lett. 2018, 121, 166801. [CrossRef]

76. Sau, J.D.; Das Sarma, S. Realizing a robust practical Majorana chain in a quantum-dot-superconductor linear array. Nat. Commun. 2012, 3, 964. [CrossRef] [PubMed]

77. Majorana, E. Teoria simmetrica dell'elettrone e del positrone. Il Nuovo Cimento (1924-1942) 1937, $14,171$. [CrossRef]

78. Ivanov, D.A. Non-Abelian Statistics of Half-Quantum Vortices in $p$-Wave Superconductors. Phys. Rev. Lett. 2001, 86, 268-271. [CrossRef] [PubMed]

79. Alicea, J.; Oreg, Y.; Refael, G.; von Oppen, F.; Fisher, M.P.A. Non-Abelian statistics and topological quantum information processing in 1D wire networks. Nat. Phys. 2011, 7, 412-417. [CrossRef]

80. Das Sarma, S.; Freedman, M.; Nayak, C. Majorana zero modes and topological quantum computation. NPJ Quantum Inf. 2015, 1, 15001. [CrossRef]

81. Bravyi, S.; Kitaev, A. Universal quantum computation with ideal Clifford gates and noisy ancillas. Phys. Rev. A 2005, 71, 022316. [CrossRef]

82. Bonderson, P.; Freedman, M.; Nayak, C. Measurement-Only Topological Quantum Computation. Phys. Rev. Lett. 2008, 101, 010501. [CrossRef]

83. Hyart, T.; van Heck, B.; Fulga, I.C.; Burrello, M.; Akhmerov, A.R.; Beenakker, C.W.J. Flux-controlled quantum computation with Majorana fermions. Phys. Rev. B 2013, 88, 035121. [CrossRef]

84. Choy, T.P.; Edge, J.M.; Akhmerov, A.R.; Beenakker, C.W.J. Majorana fermions emerging from magnetic nanoparticles on a superconductor without spin-orbit coupling. Phys. Rev. B 2011, 84, 195442. [CrossRef]

85. Nadj-Perge, S.; Drozdov, I.K.; Li, J.; Chen, H.; Jeon, S.; Seo, J.; MacDonald, A.H.; Bernevig, B.A.; Yazdani, A. Observation of Majorana fermions in ferromagnetic atomic chains on a superconductor. Science 2014, 346, 602-607. [CrossRef] [PubMed]

86. Mourik, V.; Zuo, K.; Frolov, S.M.; Plissard, S.R.; Bakkers, E.P.A.M.; Kouwenhoven, L.P. Signatures of Majorana Fermions in Hybrid Superconductor-Semiconductor Nanowire Devices. Science 2012, 336, 1003. [CrossRef] [PubMed]

87. Rokhinson, L.P.; Liu, X.; Furdyna, J.K. The fractional a.c. Josephson effect in a semiconductor-superconductor nanowire as a signature of Majorana particles. Nat. Phys. 2012, 8, 795-799. [CrossRef]

88. Laroche, D.; Bouman, D.; van Woerkom, D.J.; Proutski, A.; Murthy, C.; Pikulin, D.I.; Nayak, C.; van Gulik, R.J.J.; Nygård, J.; Krogstrup, P.; et al. Observation of the $4 \pi$-periodic Josephson effect in InAs nanowires. arXiv 2017, arXiv:1712.08459.

89. Lutchyn, R.M.; Bakkers, E.P.A.M.; Kouwenhoven, L.P.; Krogstrup, P.; Marcus, C.M.; Oreg, Y. Majorana zero modes in superconductor-semiconductor heterostructures. Nat. Rev. Mater. 2018, 3, 52-68. [CrossRef] 
90. Gangadharaiah, S.; Braunecker, B.; Simon, P.; Loss, D. Majorana Edge States in Interacting One-Dimensional Systems. Phys. Rev. Lett. 2011, 107, 036801. [CrossRef]

91. Stoudenmire, E.M.; Alicea, J.; Starykh, O.A.; Fisher, M.P. Interaction effects in topological superconducting wires supporting Majorana fermions. Phys. Rev. B 2011, 84, 014503. [CrossRef]

92. Lang, N.; Büchler, H.P. Topological states in a microscopic model of interacting fermions. Phys. Rev. B 2015, 92, 041118. [CrossRef]

93. Iemini, F.; Mazza, L.; Rossini, D.; Fazio, R.; Diehl, S. Localized Majorana-Like Modes in a Number-Conserving Setting: An Exactly Solvable Model. Phys. Rev. Lett. 2015, 115, 156402. [CrossRef] [PubMed]

94. Read, N.; Green, D. Paired states of fermions in two dimensions with breaking of parity and time-reversal symmetries and the fractional quantum Hall effect. Phys. Rev. B 2000, 61, 10267-10297. [CrossRef]

95. Fu, L.; Kane, C.L. Superconducting Proximity Effect and Majorana Fermions at the Surface of a Topological Insulator. Phys. Rev. Lett. 2008, 100, 096407. [CrossRef] [PubMed]

96. Beenakker, C.W.J. Search for Majorana Fermions in Superconductors. Annu. Rev. Condens. Matter Phys. 2013, 4, 113-136. [CrossRef]

97. Alicea, J. New directions in the pursuit of Majorana fermions in solid state systems. Rep. Prog. Phys. 2012, 75, 076501. [CrossRef] [PubMed]

98. Schuch, N.; Pérez-García, D.; Cirac, I. Classifying quantum phases using matrix product states and projected entangled pair states. Phys. Rev. B 2011, 84, 165139. [CrossRef]

99. Fidkowski, L.; Kitaev, A. Topological phases of fermions in one dimension. Phys. Rev. B 2011, 83, 075103. [CrossRef]

100. Verresen, R.; Moessner, R.; Pollmann, F. One-dimensional symmetry protected topological phases and their transitions. Phys. Rev. B 2017, 96, 165124. [CrossRef]

101. Robinson, N.J.; Altland, A.; Egger, R.; Gergs, N.M.; Li, W.; Schuricht, D.; Tsvelik, A.M.; Weichselbaum, A.; Konik, R.M. Non-Topological Majorana Zero Modes in Inhomogeneous Spin Ladders. arXiv 2018, arXiv:1806.01925.

(C) 2019 by the authors. Licensee MDPI, Basel, Switzerland. This article is an open access article distributed under the terms and conditions of the Creative Commons Attribution (CC BY) license (http:/ / creativecommons.org/licenses/by/4.0/). 